\title{
Diagnostic Yield of Mediastinal Exploration
}

\author{
Waseem Hajjar Yasser Elmedany Ahamed Bamousa Moataz Saladein \\ Mahmoud Ashour Mohammed Fouda Khaled Al-Kattan \\ Thoracic Surgery Division, Department of Surgery, King Khalid University Hospital, Riyadh, Saudi Arabia
}

\section{Key Words}

Mediastinal · Mediastenoscopy · Lymphoma •

Tuberculosis · Lymphadenopathy

\begin{abstract}
Objective: To report our experiences of surgical diagnostic procedures in patients with unidentified mediastinal pathology. Methods: From J uly 1995 to J uly 1999, 72 patients with mediastinal pathology had 73 surgical procedures for the purpose of tissue diagnosis. Of the 72 patients, 39 were female and 33 male, with an average age of 54 years. Mediastinoscopy and anterior mediastinotomy were performed in 54 and 15 patients, respectively (13 left and 2 right). Thoracoscopy was used in 3 cases, and 2 patients were diagnosed via cervical incision. Superior vena cava (SVC) obstruction was noted in 4 patients and four procedures were repeat procedures. Frozen section analysis was performed in 52 cases. Results: Tissue diagnosis was achieved in all cases, enabling a specific diagnosis in 70 cases (97\%). In 2 patients, the final diagnosis was non-specific and in 1 patient repeat biopsy was needed. The diagnoses were lymphoma ( $n=32)$, tuberculosis $(n=20)$, metastatic disease $(n=11)$ and other pathology $(n=9)$. There was no operation-related mortality although 1 patient developed
\end{abstract}

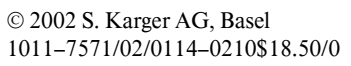

Fax + 41613061234 E-Mail karger@karger.ch www. karger.com
Accessible online at: www. karger.com/mpp mediastinal haematoma, which was treated conservatively. The 2 in-hospital deaths resulted from causes secondary to the primary disease (invasive aspergilloma or Hodgkin's lymphoma). Conclusion: Mediastinoscopy is a safe surgical procedure with high diagnostic yield. Its routine use with mediastinotomy and thoracoscopy ensures accurate diagnosis. Careful surgical technique is mandatory in repeat procedures and SVC obstruction cases.

Copyright $\odot 2002$ S. Karger AG, Base

\section{Introduction}

Carlens [1] first introduced mediastinoscopy in 1959 in an attempt to improve pre-operative staging for patients with lung cancer. Since then the procedure has become widely used to evaluate other mediastinal diseases. With modern imaging techniques and the development of advanced interventional radiology, the cause of mediastinal masses can usually be determined. Lymphoproliferative diseases of the mediastinum have become more common in the past years and differentiation from tuberculous lymphadenitis, which requires a tissue sample [2], is mandatory for proper management. When fineneedle aspiration (FNA) and true-cut needle biopsies fail, 
an open biopsy should be performed [3]. Utilising different diagnostic surgical procedures such as mediastinoscopy and anterior mediastinotomy ensures accurate diagnosis and avoids major surgical exploration such as thoracotomy or sternotomy [4]. In this paper, we review a 4-year experience of surgical procedures to diagnose patients presenting with unidentified mediastinal diseases. These procedures, their techniques and outcomes are discussed and evaluated.

\section{Subjects and Methods}

Over a period of 4 years (July 1995 to July 1999), 72 patients with undiagnosed mediastinal pathology were referred for tissue biopsy to obtain a tissue diagnosis. There were 39 females and 33 males, with an average age of 54 years (ranging from 6 to 90 years). Nine patients were asymptomatic at presentation and were diagnosed by abnormal chest radiography. Cough, present in 44 , and haemoptysis in 11 were the most common presenting symptoms. Four patients presented with superior vena cava (SVC) obstruction. Four other patients were referred from other hospitals after previous mediastinoscopy had been performed without definitive diagnosis. At the time of surgery, 12 patients were on steroid treatment for SVC obstruction in 4 and empirically in 8. Eight patients were on empirical antituberculous treatment for a mean duration of 4 months (range 2-6 months), and as the disease progressed they were referred for tissue diagnosis. Four patients had previous chemotherapy for a diagnosis of lymphoma. However, a second biopsy was needed to either confirm the diagnosis in 2 non-responders or to rebiopsy after recurrence in 2 patients with initial good response.

All procedures were performed in either King Khalid University Hospital $(n=45)$ or King Faisal Specialist Hospital and Research Centre $(n=27)$ in Riyadh. All patients with mediastinal diseases were initially evaluated with a computed tomography (CT) scan. Additional procedures such as bronchoscopy with broncho-alveolar lavage or transbronchial biopsy were also performed in 15 patients. FNA biopsy or true-cut biopsy was occasionally performed in 12 patients. When diagnosis was not achieved, the patient was referred to the Thoracic Surgery Unit for further evaluation. Cervical mediastinoscopy (Karl Storz-Endoscope, Tuttlingen, Germany) was performed in 54 cases in which the patients had anterior mediastinal mass or lymphoproliferative disease involving the paratracheal, tracheo-bronchial or subcarinal groups of lymph nodes, while anterior mediastinotomy was performed in 15 cases in which the disease was either on one side of the anterior mediastinum or the lymph node involved belonged to the para-aortic and subaortic group. The latter was on the left side in 13 cases and the right side in 2 .

Thoracoscopy was used in 3 cases ( 2 right and 1 left) to detect the presence of inferior mediastinal disease and the involvement of either para-oesophageal or the inferior pulmonary ligament node areas. In the remaining 2 cases, cervical incision before mediastinoscopy revealed and accessible lesion in the deep cervical area in 1 and in the supraclavicular area in another.

All procedures were performed using general anaesthesia, and rigid bronchoscopy preceded the procedure in 32 cases. In all procedures and in mediastinoscopy in particular, multiple biopsies from different areas were taken. Fresh samples were sent for frozen section

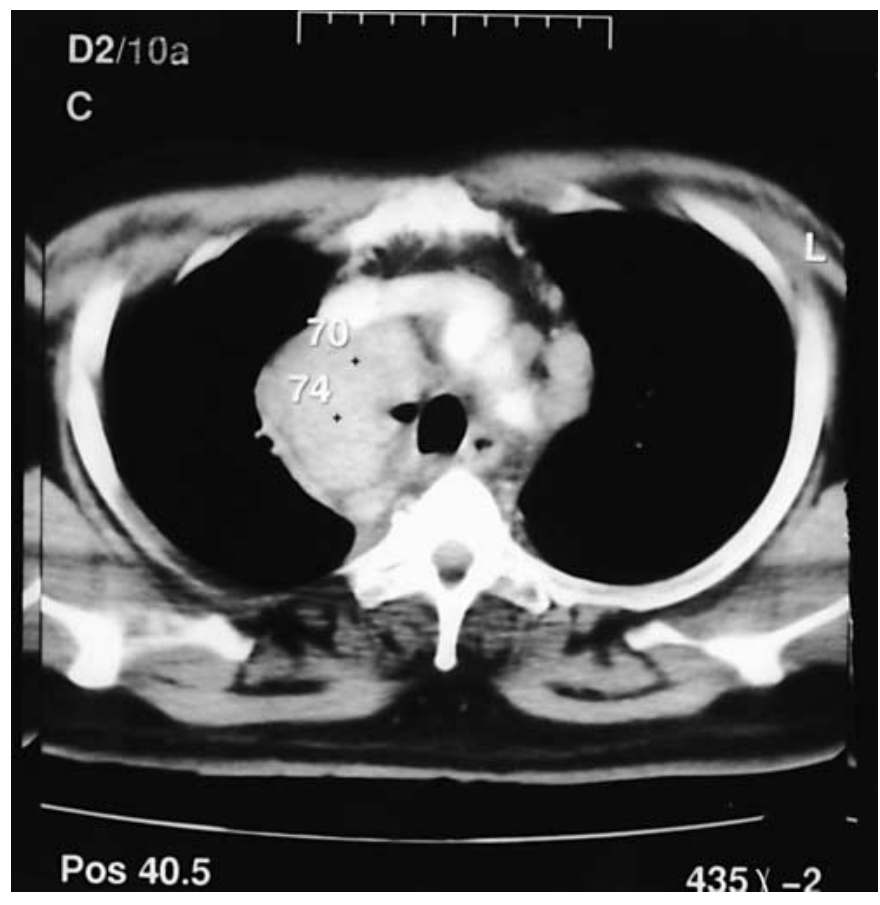

Fig. 1. CT scan of 39-year-old man demonstrating large paratracheal mediastinal lymphadenopathy. Biopsy showed granulomatous lymphadenitis with caseous necrosis, tuberculosis.

examination to confirm whether the biopsy was adequate and diagnostic before closure (when tuberculosis was not suspected). In 19 cases in which previous mediastinal biopsy was inadequate, a decision was made to either perform the second biopsy through a second approach or redo the procedure through the previous approach.

Samples from all the 72 patients were also sent to microbiology to exclude tuberculosis and for culture for possible bacterial or fungal diseases. The presence of evidence of SVC obstruction did not contra-indicate mediastinal exploration, which was meticulously performed to prevent excessive bleeding. Complete follow-up based on the immediate results of these diagnostic procedures was available for all patients.

\section{Results}

Tissue diagnosis was achieved in all patients with specific diagnosis, in 70 patients $(97 \%)$. The diagnoses were lymphoma $(n=32)$, tuberculosis $(n=20)$, metastatic cancer $(n=11)$ and other pathology $(n=11)$. Histological diagnosis was non-specific in 2 cases, 1 of which was due to inadequate biopsy. A repeat procedure was performed and showed lymphoma. The mean follow-up period was 4 months. There was no operation-related mortality. However, there were 2 in-hospital deaths secondary to the pri- 

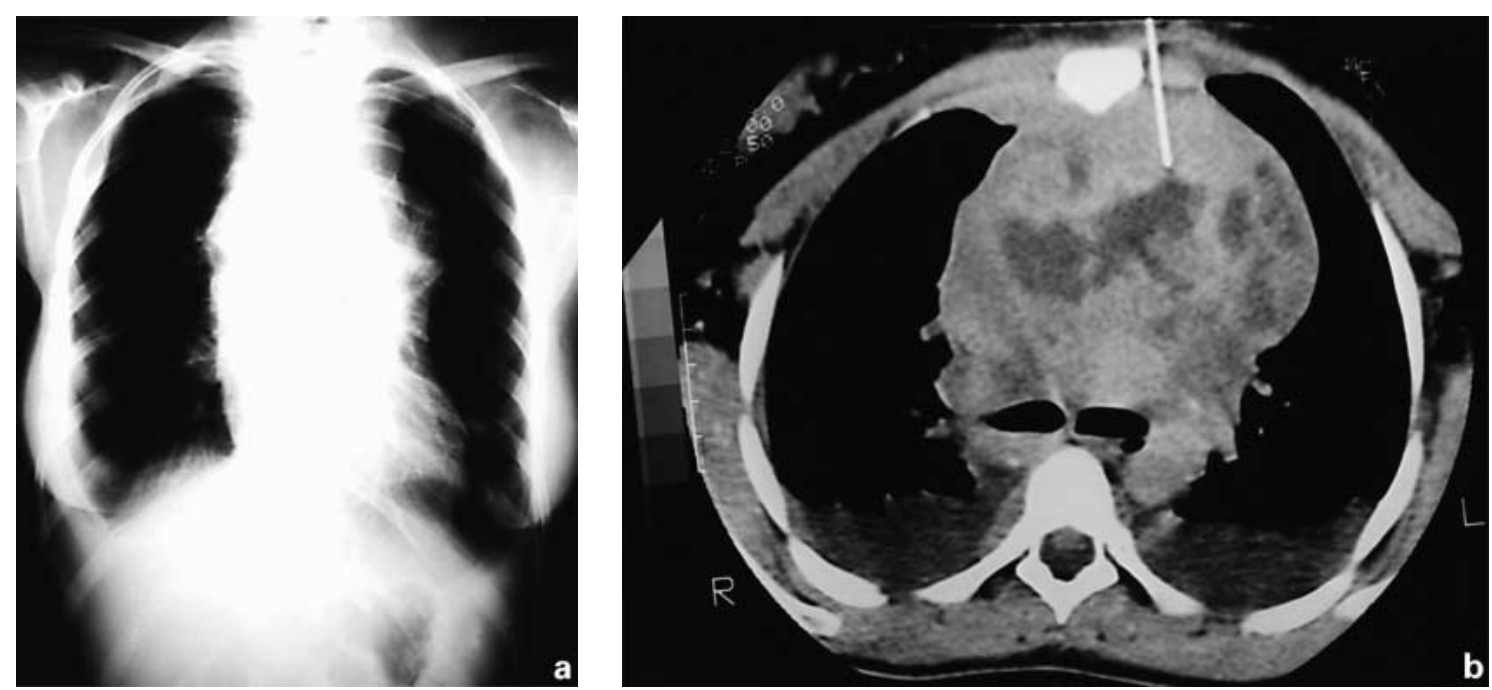

Fig. 2. a Chest radiograph of a 15 -year-old girl demonstrating large mediastinal lymphadenopathy. b CT scan revealing the anterior mediastinal mass during the true-cut biopsy which showed T-cell lymphoma.

mary disease, invasive aspergilloma and Hodgkin's lymphoma. Intra-operatively, excess venous bleeding was noted in 5 patients who responded to compression in the mediastinoscopy plane. One patient developed postoperative anterior mediastinal haematoma, which was stable and treated conservatively without surgical exploration. Histological results before closure were available in 60 cases.

\section{Discussion}

Mediastinal diseases are a common presentation of a variety of pathological disorders. The introduction of mediastinoscopy has provided the highest possible diagnostic accuracy. It can be performed with low risk and a short hospital stay [5]. Moreover, the diagnostic yield can be improved with the availability of thoracoscopy and anterior mediastinotomy in selected cases [6]. Empirical therapy, especially in cases in which tuberculosis is suspected, should no longer be applied. When lymphoproliferative diseases are suspected, tissue diagnosis is warranted not only to confirm the diagnosis but also to identify the histologic subtypes where different therapies and prognoses vary from one type to another.

Although mediastinal diseases vary in their presentation, from being asymptomatic to presenting SVC obstruction, cough remains the commonest presenting symptom either due to compression effects of the upper airway or underlying pulmonary disease [7] as was seen in 44 of 72 patients in this study. Careful history and complete physical examination are the bases of proper diagnosis. In addition, utilisation of different radiological investigations should be guided with the clinical diagnosis.

Chest radiography indicates the mediastinal widening and a lateral view helps to allocate the anatomical position of the lesion, while CT scan of the chest and mediastinum, especially with contrast, enables detailed interpretation of these lesions [8]. Magnetic resonance imaging (MRI) has limited additional diagnostic value over the CT scan [9]. In our series, CT scan was performed for all patients; MRI was performed in only 3 patients to exclude spinal involvement.

In the presence of suspected tuberculosis or other pulmonary diseases such as sarcoidosis, thorough investigation should be made, including bronchoscopy with broncho-alveolar lavage with or without biopsy. With good histopathology facilities, an attempt at CT-guided biopsy is a less invasive diagnostic technique with high diagnostic yield. A specimen can be obtained with an FNA or true-cut biopsy. The latter carries a higher yield but with higher complication rate and is not suitable in all cases [10]. Mediastinoscopy remains the preferred tool of tissue diagnosis in evaluation of anterior mediastinal masses. Video-assisted or not, a full exploration of the mediastinal lesion should be made, guided by the radiological and clinical picture [10]. Adequate biopsy is required and a frozen section examination is better done to determine 
whether sufficient tissue has been obtained to achieve a firm final diagnosis. When tuberculosis is suspected, most laboratories avoid frozen sections to prevent cross-infection.

When the patient presents with SVC obstruction, mediastinoscopy is considered dangerous due to the increased risk of bleeding and the associated risks of general anaesthesia [11]. The latter includes hypotension and airway collapse. Since a malignant aetiology was reported in $95 \%$ of the cases, the use of empirical chemotherapy or radiotherapy is inappropriate. In addition, subsequent histological diagnosis may prove to be difficult. Careful mediastinoscopy by an experienced surgical team and well-equipped facility is essential to ensure the safety of the procedure. A redo procedure is equally difficult and if no other access can provide a diagnostic yield, careful dissection is warranted.

Alternatively, mediastinal exploration can also be achieved in selected cases by anterior mediastinotomy, which provides a satisfactory exploration especially to the para-aortic and subaortic groups of lymph nodes on the left side. The para-oesophageal and lower para-aortic lesions including the inferior pulmonary ligament nodes are best approached via thoracoscopy [12]. The latter can also provide an accurate evaluation of operability in surgically treated cases. We have a high incidence of tuberculosis in comparison to data from the European reports. This incidence is almost equal in the diagnosis of lymphoma in our series [10]. Although the CT scan can suggest the underlying pathology, tissue diagnosis remains obligatory.

In our study, these procedures proved to be excellent in terms of achieving a definitive diagnosis and in the safety of the procedure. Diagnosis was achieved in $97 \%$ of the cases and a further biopsy elevated this figure to $100 \%$. There were no operative deaths and morbidity was limited to 1 case of postoperative haematoma that did not require re-exploration or blood transfusion. In our opinion, mediastinoscopy is a useful diagnostic tool with minimal risk. In all cases of mediastinal pathology once initially diagnosed, histological confirmation should be available before starting treatment. If less invasive procedures fail to achieve tissue diagnosis, mediastinoscopy should be performed. The presence of SVC obstruction or previous biopsy are not absolute contra-indications. The utilisation of thoracoscopy or anterior mediastinal approach will increase the diagnostic yield in selected cases.

\section{Conclusion}

We conclude that mediastinoscopy is a safe invasive procedure with high diagnostic yield in different mediastinal pathology. Its routine use with mediastinostomy and thoracoscopy insured accurate diagnosis. Careful mediastinoscopic technique is mandatory especially in repeat intervention and SVC obstruction cases.

\section{References}

1 Carlens E: Mediastinoscopy: A method for inspection and tissue biopsy in the superior mediastinum. Dis Chest 1959;36:343-347.

2 Ginsberg RJ: Evaluation of the mediastinum by invasive techniques. Surg Clin North Am 1987;67:1025-1035

3 Ellison E, Laquerta P, Martin SE: Fine needle aspiration diagnosis of mycobacterial lymphadenitis: Sensitivity and predictive value in the United States. Acta Cytol 1999;43:153157.

4 Goldstraw P: Mediastinal exploration by mediastinoscopy and mediastinotomy. Br $\mathrm{J}$ Dis Chest 1988;82:111-120.
5 Jolly PC, Li W, Anderson RP: Anterior and cervical mediastinoscopy for determining operability and predicting respectability in lung cancer. J Thorac Cardiovasc Surg 1980;79: 366-371.

6 Landreneau RJ, Dowling RD, Castillo WM, Ferson PF: Thoracoscopic resection of an anterior mediastinal tumor. Ann Thorac Surg 1992; 54:142-144.

7 Parish JM, Marschke RF, Dines DE, Lee RE: Etiological consideration in superior vena cava syndrome. Mayo Clin Proc 1981;56:407-413.

8 Kushihashi T, Munechika H, Motoya H, Hamada K, Satoh I, Naitoh H, Nakajima H, Soejima K: CT and MR finding in tuberculous mediastinitis. J Comput Assist Tomogr 1995; 19:379-382.
9 Moon WK, Im JG, Yu IK, Lee SK, Yeon KM, Han MC: Mediastinal tuberculosis lymphadenitis: MR imaging appearance with clinicopathologic correlation. AJR Am Roentgenol 1996;166:21-25.

10 Khan J, Akhtar M, Von Sinner WN, Bouchama A, Bazarbashi M: CT-guided fine needle aspiration biopsy in the diagnosis of mediastinal tuberculosis. Chest 1994;106:1329-1332.

11 Jahangiri J, Goldstraw P: The role of mediastinoscopy in superior vena caval obstruction. Ann Thorac Surg 1995;59:453-455.

12 Hutter J, Junger W, Miller K, Moritz E: Subxiphoidal videomediastinoscopy for diagnostic acceess to the anterior mediastinum. Ann Thorac Surg 1998;66:1427-1428. 